\title{
New results on soft particle production in heavy-ion collisions with ATLAS
}

\author{
Adam Trzupek, on behalf of the ATLAS Collaboration* \\ Institute of Nuclear Physics Polish Academy of Sciences \\ ul. Radzikowskiego 152 \\ 31-342 Kraków, Poland \\ E-mail: Adam.Trzupekeifj.edu.pl
}

\begin{abstract}
The measurement of flow $v_{2}-v_{5}$ harmonics in Xe+Xe collisions at the energy of $\sqrt{s_{\mathrm{NN}}}=5.44 \mathrm{TeV}$ were recently performed in the ATLAS experiment at the LHC. Results on the $p_{\mathrm{T}}$ and centrality dependence of $v_{\mathrm{n}}$ harmonics are presented. A comparison of the measured $v_{\mathrm{n}}$ to $\mathrm{Pb}+\mathrm{Pb}$ results and theoretical predictions is shown. The data indicate stronger event-to-event fluctuations of $v_{\mathrm{n}}$ harmonics in the $\mathrm{Xe}+\mathrm{Xe}$ collision system as compared to $\mathrm{Pb}+\mathrm{Pb}$ collisions. For $\mathrm{Pb}+\mathrm{Pb}$ collisions at the energy of $\sqrt{s_{\mathrm{NN}}}=5.02 \mathrm{TeV}$, the modified Pearson's correlation coefficient for the event-wise mean transverse momentum and the square of the magnitude of flow harmonics is also presented. Significant, non-zero correlation coefficients for $v_{2}, v_{3}$ and $v_{4}$ harmonics are obtained. The coefficients weakly depend on the transverse momentum range of the selected particles. The coefficient for the second order harmonic is negative in peripheral events, has a significant positive value for mid-central events and drops in the most central collisions. Measured Pearson's coefficients are compared to model predictions.
\end{abstract}

Sixth Annual Conference on Large Hadron Collider Physics (LHCP2018)

4-9 June 2018

Bologna, Italy

\footnotetext{
*Speaker.
} 


\section{Introduction}

Experimental results and theoretical predictions indicate that the collective flow of particles produced in heavy-ion collisions is related to the anisotropy of the initial geometry of the collision zone, resulting in anisotropic pressure gradients building up in the Quark Gluon Plasma (QGP). In the framework of hydrodynamical models, the properties of the produced medium were found to be similar to those of a nearly perfect fluid characterised by a very low ratio of viscosity to entropy density [1]. The azimuthal angle distributions of produced particles in heavy-ion collisions are characterised by a Fourier series [2]:

$$
\frac{\mathrm{d} N}{\mathrm{~d} \phi}=\frac{N_{0}}{2 \pi}\left(1+\sum_{\mathrm{n}=1} 2 v_{\mathrm{n}} \cos \left[\mathrm{n}\left(\phi-\Phi_{\mathrm{n}}\right)\right]\right),
$$

where $\phi$ is the azimuthal angle of the particle, and the $v_{\mathrm{n}}$ and $\Phi_{\mathrm{n}}$ are the flow harmonics and the reaction plane angles of $\mathrm{n}^{\text {th }}$-order anisotropy. The second harmonic, called elliptic flow $\left(v_{2}\right)$, and higher-order flow harmonics characterise the "elliptical" shape of the initial interaction region and its higher modes, respectively.

Recently in ATLAS, the azimuthal anisotropy was studied using a complementary sample of $\mathrm{Xe}+\mathrm{Xe}$ collisions at a centre-of-mass energy per nucleon pair of $\sqrt{s_{\mathrm{NN}}}=5.44 \mathrm{TeV}$ to bridge the gap between small $(p p$ and $p+\mathrm{Pb})$ and large $(\mathrm{Pb}+\mathrm{Pb})$ collisions at the LHC [3]. The new results on $v_{\mathrm{n}}$ harmonics obtained with the multi-particle correlation and scalar product methods in $\mathrm{Xe}+\mathrm{Xe}$ collisions are discussed in Section 2.

The properties of the QGP are also studied with measurements of correlations between flow harmonics of different order [4], analyses of event shapes as well as the event-to-event correlations of flow harmonics and the mean transverse momentum of produced particles per event [5]. The strength of the $v_{\mathrm{n}}$-mean $p_{\mathrm{T}}$ correlations in $\mathrm{Pb}+\mathrm{Pb}$ collisions at the energy $\sqrt{s_{\mathrm{NN}}}=5.02 \mathrm{TeV}$, quantified by the modified Pearson's correlation coefficient [6], is presented in Section 3.

\section{Azimuthal anisotropy in $\mathrm{Xe}+\mathrm{Xe}$ interactions}

The measurement of azimuthal anisotropy in $\mathrm{Xe}+\mathrm{Xe}$ interactions at $\sqrt{s_{\mathrm{NN}}}=5.44$ is based on the data sample of integrated luminosity of $3 \mu \mathrm{b}^{-1}$ collected by the ATLAS experiment in October 2017 [3]. The analysis uses charged particles of transverse momentum $p_{\mathrm{T}}>0.5 \mathrm{GeV}$ within the pseudorapidity range $|\eta|<2.5$, detected in the ATLAS inner detector [7]. The flow harmonics $v_{\mathrm{n}}$, $\mathrm{n}=2-5$ measured by the scalar product (SP) method [8] are shown in Figure 1 for $0-5 \%, 20-30 \%$ and $50-60 \%$ centrality intervals. A similar $p_{\mathrm{T}}$-dependence is observed for the flow harmonics in $\mathrm{Xe}+\mathrm{Xe}$ as it was measured for $\mathrm{Pb}+\mathrm{Pb}$ collisions: a rise up to $p_{\mathrm{T}} \approx 3 \mathrm{GeV}$ and then decrease with $p_{\mathrm{T}}$. The $v_{2}$ dominates at all centralities, except for the $0-5 \%$ most central collisions where, at intermediate $p_{\mathrm{T}}, v_{3}$ becomes larger than $v_{2}$, indicating that the dominant source of observed flow comes from the initial geometry fluctuations.

The comparison of the $v_{\mathrm{n}}$ in $\mathrm{Xe}+\mathrm{Xe}$ and $\mathrm{Pb}+\mathrm{Pb}$ collisions, measured with the two-particle correlation (2PC) method [2] is shown in Figure 2 as a function of the collision centrality. The ratios of the $\mathrm{Xe}+\mathrm{Xe} v_{\mathrm{n}}$ to the $\mathrm{Pb}+\mathrm{Pb} v_{\mathrm{n}}$ are also shown. Generally, the ratio is larger than unity in central collisions and decreases to values smaller than 1 towards more peripheral events. In particular, the ratio for $v_{2}$ is reaching 1.4 within $0-5 \%$ centrality interval and becomes roughly 0.9 at the $60-70 \%$ centrality. For $v_{3}$, the ratio is decreasing from about 1.2 to 0.9 over the $0-60 \%$ 

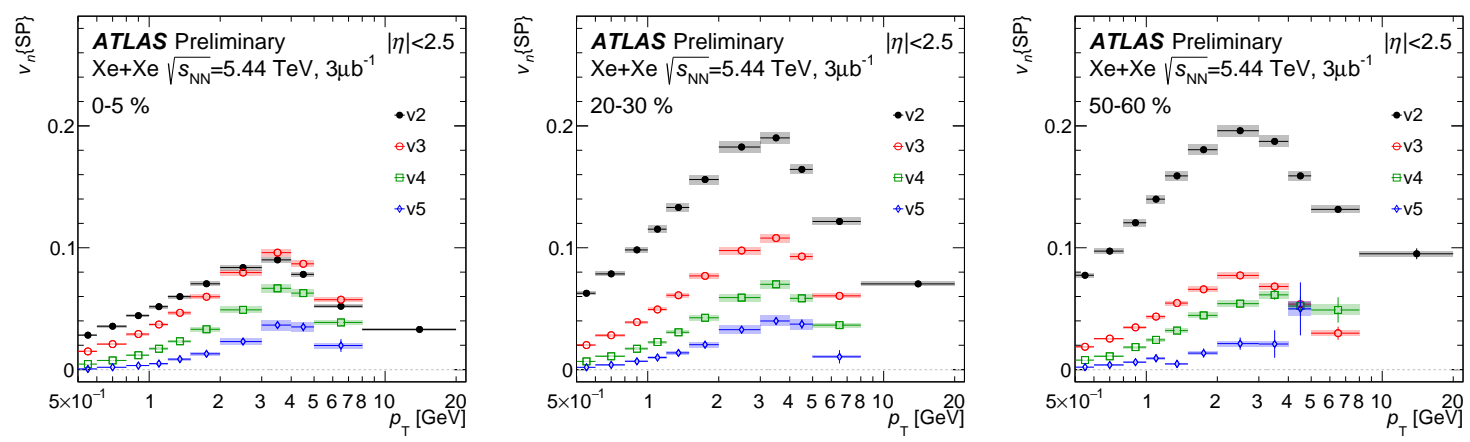

Figure 1: Flow harmonics $v_{2}-v_{5}$ measured with the SP method as a function of $p_{\mathrm{T}}$ in centrality intervals: 0-5\% (left), 20-30\% (middle) and 50-60\% (right) [3].
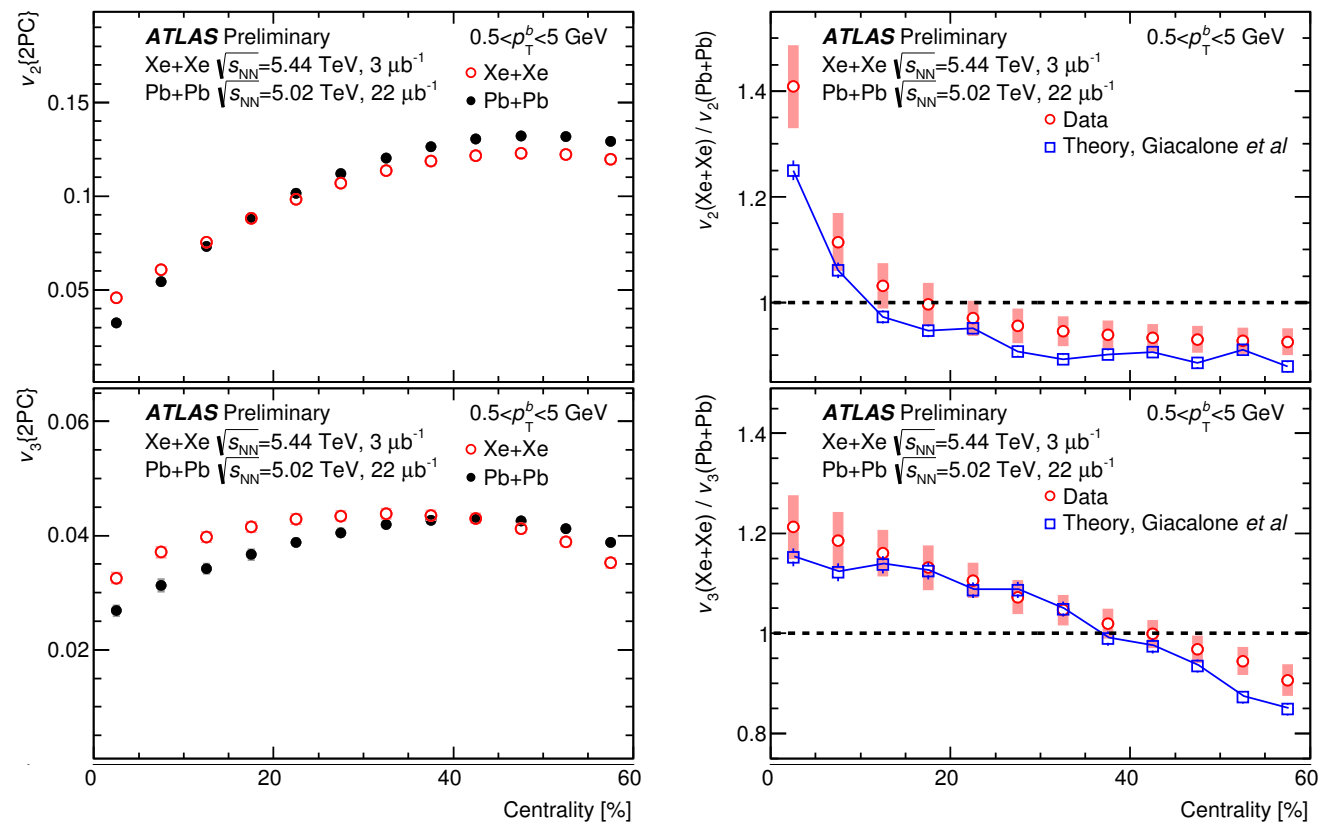

Figure 2: Left panels: comparisons of the centrality dependence of the $v_{2}$ (top) and $v_{3}$ (bottom) measured using the $2 \mathrm{PC}$ method in $\mathrm{Pb}+\mathrm{Pb}$ and $\mathrm{Xe}+\mathrm{Xe}$ collisions. Right panels: the ratios $v_{\mathrm{n}}(\mathrm{Xe}+\mathrm{Xe}) / v_{\mathrm{n}}(\mathrm{Pb}+\mathrm{Pb})[3]$. The ratios are compared to theoretical predictions [9].
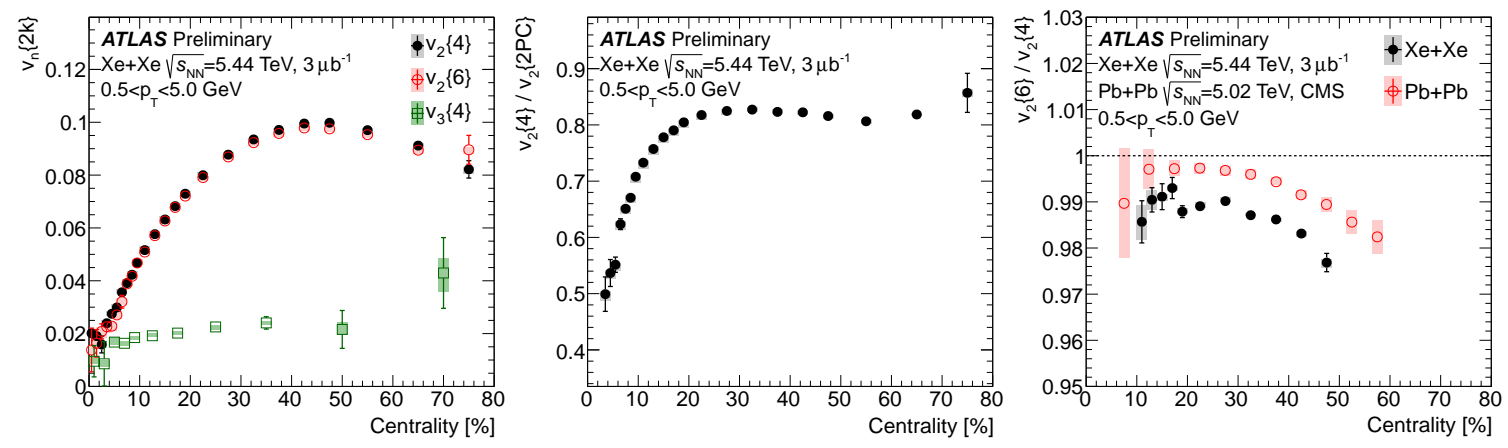

Figure 3: Flow harmonics obtained with four-particle $\left(v_{2}\{4\}, v_{3}\{4\}\right)$ and six-particle $\left(v_{2}\{6\}\right)$ cumulants (left panel) and the ratio $v_{2}\{4\} / v_{2}\{2 P C\}$ (middle panel) as a function of centrality of $\mathrm{Xe}+\mathrm{Xe}$ collisions. The ratio $v_{2}\{6\} / v_{2}\{4\}$ (right panel) for $\mathrm{Xe}+\mathrm{Xe}$ and $\mathrm{Pb}+\mathrm{Pb}$ results [3]. 
centrality range. A good qualitative agreement between the ratios for $v_{2}$ and $v_{3}$ harmonics and theoretical predictions [9] is observed.

To quantify the type and strength of flow fluctuations, $2 k$-particle cumulants, calculated for charged particles with $0.5<p_{\mathrm{T}}<5.0 \mathrm{GeV}$, are used to obtain $v_{\mathrm{n}}\{2 k\}, k=1,2,3$. The centrality dependence of $v_{2}$ harmonics for different orders of cumulants is shown in the left panel of Figure 3. Similar magnitudes of $v_{2}\{4\}$ and $v_{2}\{6\}$ harmonics indicate that $v_{2}$ fluctuations are close to Gaussian. Ratios $v_{2}\{4\} / v_{2}\{2 P C\}$ and $v_{2}\{6\} / v_{2}\{4\}$ are shown in the middle and right panels of Figure 3, respectively. For a Gaussian fluctuation model, the ratio between $v_{2}\{4\}$ and $v_{2}\{2 P C\}$ reflects the relative strength of flow fluctuations. The results for $v_{2}\{4\} / v_{2}\{2 P C\}$ indicate that flow fluctuations are the largest in the most central collisions. The ratio $v_{2}\{6\} / v_{2}\{4\}$ is expected to be unity for the Gaussian model of flow fluctuations. Indeed, for both collision systems the ratio is not significantly different than 1 , indicating that Gaussian fluctuations dominate and the nonGaussian contribution is very small. Comparison of this ratio measured in $\mathrm{Pb}+\mathrm{Pb}$ and $\mathrm{Xe}+\mathrm{Xe}$ collisions suggests that the non-Gaussian component in $\mathrm{Xe}+\mathrm{Xe}$ is slightly larger than in $\mathrm{Pb}+\mathrm{Pb}$ for mid-central collisions.

The study of symmetric and asymmetric cumulants turns out to be a useful constraint on hydrodynamic models of the dense and hot medium created in heavy-ion collisions [10]. The symmetric, $\mathrm{sc}_{\mathrm{m}, \mathrm{n}}\{4\}$, and asymmetric, $\mathrm{ac}_{\mathrm{n}, 2 \mathrm{n}}\{3\}$, cumulants are defined as:

$$
\begin{aligned}
\operatorname{sc}_{\mathrm{m}, \mathrm{n}}\{4\} & =\left\langle\operatorname{corr}_{\mathrm{m}, \mathrm{n}}\{4\}\right\rangle-\left\langle\operatorname{corr}_{m}\{2\}\right\rangle\left\langle\operatorname{corr}_{n}\{2\}\right\rangle, \\
\operatorname{ac}_{\mathrm{n}, 2 \mathrm{n}}\{3\} & =\left\langle\operatorname{corr}_{\mathrm{n}, 2 \mathrm{n}}\{3\}\right\rangle
\end{aligned}
$$

where $\operatorname{corr}_{n}\{2\}$ and $\operatorname{corr}_{n}\{4\}$ stand for the two- and four-particle azimuthal correlations, $\operatorname{corr}_{\mathrm{m}, \mathrm{n}}\{4\}$ represents the correlation of two flow harmonics of different orders $m$ and $n$ [3] and the three-particle correlation $\operatorname{corr}_{n, 2 n}\{3\}$ is related to correlations of the event plane

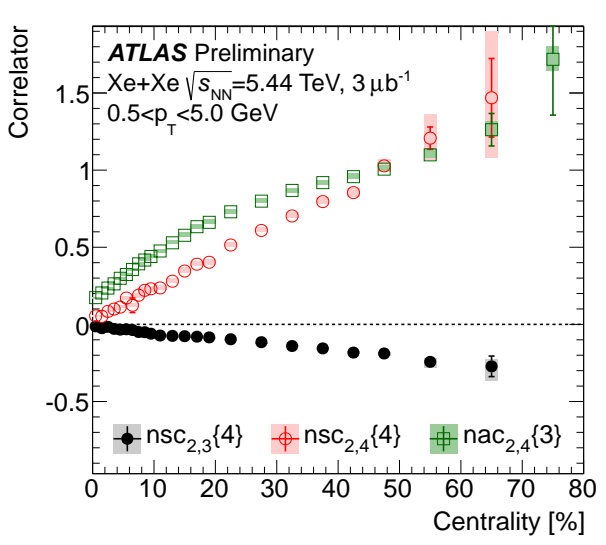

Figure 4: Normalized symmetric and asymmetric cumulants as a function of centrality [3]. angles $\Psi_{n}$ and $\Psi_{2 n}[11,12]$. The averaging over events is denoted by $\langle\ldots\rangle$. To remove the dependence on the magnitude of flow harmonics the normalized symmetric and asymmetric cumulants are obtained:

$$
\mathrm{nsc}_{\mathrm{m}, \mathrm{n}}\{4\}=\frac{\mathrm{sc}_{\mathrm{m}, \mathrm{n}}\{4\}}{\left\langle v_{\mathrm{m}}^{2}\right\rangle\left\langle v_{\mathrm{n}}^{2}\right\rangle}, \quad \operatorname{nac}_{\mathrm{n}, 2 \mathrm{n}}\{3\}=\frac{\mathrm{ac}_{\mathrm{n}, 2 \mathrm{n}}\{3\}}{\sqrt{\left\langle v_{\mathrm{n}}^{4}\right\rangle\left\langle v_{2 \mathrm{n}}^{2}\right\rangle}}
$$

where $\left\langle v_{\mathrm{n}}^{2}\right\rangle$ are calculated using the $2 \mathrm{PC}$ method and $\left\langle v_{\mathrm{n}}^{4}\right\rangle=\left\langle\operatorname{corr}_{n}\{4\}\right\rangle$. Figure 4 shows the centrality dependence of normalized symmetric and asymmetric cumulants measured in $\mathrm{Xe}+\mathrm{Xe}$ collisions. A negative $\mathrm{nsc}_{2,3}\{4\}$ is observed over the entire centrality range, indicating that $v_{2}$ and $v_{3}$ are anticorrelated. The positive $\mathrm{nsc}_{2,4}\{4\}$ reflects the correlation between the non-linear hydrodynamic response of $v_{2}$ and the linear component of $v_{4}$. The asymmetric cumulant nac ${ }_{2,4}\{3\}$ shows a similar centrality dependence as $\mathrm{nsc}_{2,4}\{4\}$, but with a larger magnitude. The correlations are very weak in central collisions, but increase rapidly towards peripheral collisions. 

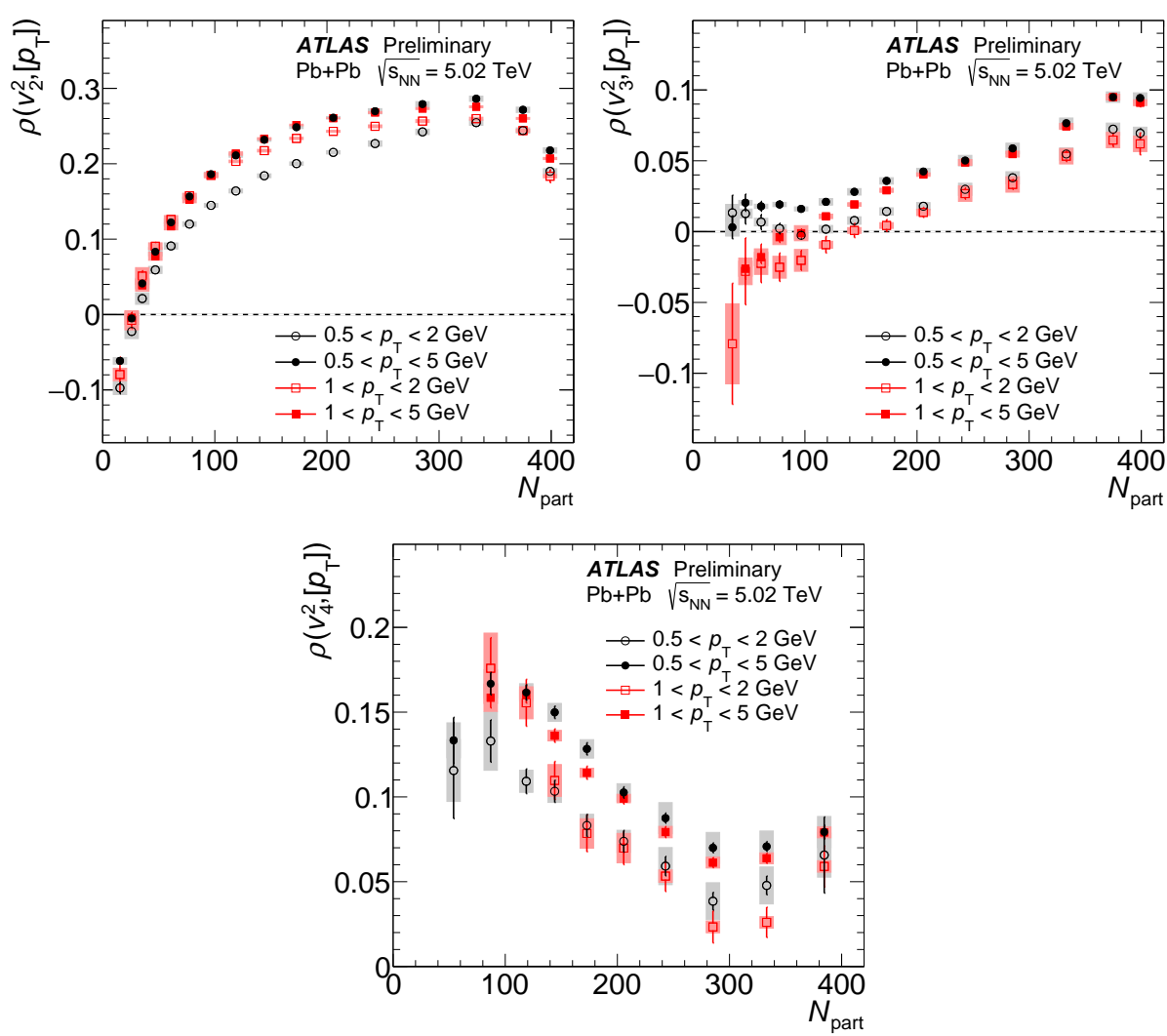

Figure 5: The modified Pearson's coefficient, $\rho\left(v_{\mathrm{n}}^{2},\left[p_{\mathrm{T}}\right]\right), \mathrm{n}=2$ (top left), 3 (top right) ,4 (bottom) as a function of $N_{\text {part }}$ in $5.02 \mathrm{TeV} \mathrm{Pb}+\mathrm{Pb}$ collisions for different $p_{\mathrm{T}}$ intervals [6].

\section{The $v_{\mathbf{n}}-$ mean $p_{\mathbf{T}}$ correlations in $\mathbf{P b}+\mathbf{P b}$ collisions}

The modified Pearson's $\rho$ coefficient can be used to measure the strength of the $v_{\mathrm{n}}-\left[p_{\mathrm{T}}\right]$ correlation [13], where $\left[p_{T}\right]$ denotes the mean transverse momentum of charged particles in an event. It is defined as:

$$
\left.\rho\left(v_{\mathrm{n}}^{2},\left[p_{\mathrm{T}}\right]\right)\right)=\frac{\operatorname{cov}\left(v_{\mathrm{n}}\{2\}^{2},\left[p_{\mathrm{T}}\right]\right)}{\sqrt{\operatorname{Var}\left(v_{\mathrm{n}}\{2\}^{2}\right)_{\mathrm{dyn}}} \sqrt{c_{k}}},
$$

where in the numerator the covariance between the $v_{n}\{2\}^{2}$ and $\left[p_{\mathrm{T}}\right]$ is used. To suppress non-flow effects, the $v_{n}\{2\}^{2}$ values are obtained using sub-events separated by 1.5 unit in pseudorpidity, $|\eta|>0.75$, while the event mean transverse momentum, $\left[p_{\mathrm{T}}\right]$, is obtained using charged particles with $|\eta|<0.5$ and in different $p_{\mathrm{T}}$ ranges. The dynamical variance of $v_{n}\{2\}^{2}$ is defined as [14]:

$$
\operatorname{Var}\left(v_{n}^{2}\right)_{\mathrm{dyn}}=v_{n}\{2\}^{4}-v_{n}\{4\}^{4}=\left\langle\operatorname{corr}_{n}\{4\}\right\rangle-\left\langle\operatorname{corr}_{n}\{2\}\right\rangle^{2},
$$

where $\operatorname{corr}_{n}\{2\}$ and $\operatorname{corr}_{n}\{4\}$ are two- and four-particle correlations and angular brackets represent averaging over events [11]. The $\left[p_{\mathrm{T}}\right]$ variance is calculated by the dynamical $p_{\mathrm{T}}$ fluctuation magnitude $[15,16], c_{k}$, defined as:

$$
c_{k}=\left\langle\frac{1}{N_{\text {pair }}} \sum_{i} \sum_{j \neq i}\left(p_{\mathrm{T}, i}-\left\langle\left[p_{\mathrm{T}}\right]\right\rangle\right)\left(p_{\mathrm{T}, j}-\left\langle\left[p_{\mathrm{T}}\right]\right\rangle\right)\right\rangle .
$$

where $N_{\text {pair }}$ denotes the number of particle pairs in an event. 
The measurement of the $\rho$ coefficient [6] is done for the $5.02 \mathrm{TeV}$ minimum-bias $\mathrm{Pb}+\mathrm{Pb}$ data sample with the integrated luminosity of $22 \mu \mathrm{b}^{-1}$. The measured $c_{k}, \operatorname{Var}\left(v_{n}^{2}\right)_{\text {dyn }}$ and covariance values are combined to form the modified Pearson's coefficient $\rho\left(v_{\mathrm{n}}^{2},\left[p_{\mathrm{T}}\right]\right)$, for $n=2-4$. Figure 5 shows $\rho\left(v_{\mathrm{n}}^{2},\left[p_{\mathrm{T}}\right]\right)$ as a function of centrality expressed by the average number of nucleons participating in the $\mathrm{Pb}+\mathrm{Pb}$ collision, $N_{\text {part }}$. For all $p_{\mathrm{T}}$ intervals, $\rho\left(v_{2}^{2},\left[p_{\mathrm{T}}\right]\right)$ rapidly increases with collision centrality for $N_{\text {part }}<100$ starting from negative values at $N_{\text {part }}<40$. A weaker increase of the correlation coefficient is observed over the $N_{\text {part }}$ range of 100-350. The strongest correlations, $\rho\left(v_{2}^{2},\left[p_{\mathrm{T}}\right]\right)=0.24-0.30$ are observed at $N_{\text {part }} \sim 320$, and then $\rho\left(v_{2}^{2},\left[p_{\mathrm{T}}\right]\right)$ decreases towards more central collisions. The significant correlation observed for mid-central events is attributed to a stronger hydrodynamic response to the large initial state eccentricities [17]. The correlation coefficient values calculated with the upper $p_{\mathrm{T}}$ limit of $2 \mathrm{GeV}$ are $10-20 \%$ smaller than the values obtained with $p_{\mathrm{T}}$ limit of $5 \mathrm{GeV}$.

The correlation coefficient $\rho\left(v_{3}^{2},\left[p_{\mathrm{T}}\right]\right)$ is also measured. The results, displayed in Figure 5, show that $\rho\left(v_{3}^{2},\left[p_{\mathrm{T}}\right]\right)$ is smaller and has a weaker centrality dependence compared to $\rho\left(v_{2}^{2},\left[p_{\mathrm{T}}\right]\right)$. It is positive for all measured centralities, except for $N_{\text {part }}<100$ where it is negative or consistent with 0 . The correlations for $p_{\mathrm{T}}$ ranges with the same maximum $p_{\mathrm{T}}$ are consistent with each other for $N_{\text {part }}>100$ and the values obtained with the higher maximum $p_{\mathrm{T}}$ threshold are larger.

The correlation coefficient $\rho\left(v_{4}^{2},\left[p_{\mathrm{T}}\right]\right)$ is also evaluated for the same four $p_{\mathrm{T}}$ ranges. Significant positive correlations are observed over the full $N_{\text {part }}$ range (Fig. 5, bottom panel). At moderate and large $N_{\text {part }}$, the fourth order correlations are weaker than those for the elliptic flow. The largest values of $\rho\left(v_{4}^{2},\left[p_{\mathrm{T}}\right]\right)$ are observed at low $N_{\text {part }} \approx 100$. For more central collisions $\rho\left(v_{4}^{2},\left[p_{\mathrm{T}}\right]\right)$ decreases with $N_{\text {part }}$ up to about $N_{\text {part }} \approx 300$, and rises slowly at higher values. The magnitude of the $\rho\left(v_{3}^{2},\left[p_{\mathrm{T}}\right]\right)$ and $\rho\left(v_{4}^{2},\left[p_{\mathrm{T}}\right]\right)$ correlations is similar for $N_{\text {part }}>270$.

In Figure 6 predictions for $\rho\left(v_{2}^{2},\left[p_{\mathrm{T}}\right]\right)$ and $\rho\left(v_{3}^{2},\left[p_{\mathrm{T}}\right]\right)$ as a function of $N_{\text {part }}$ are compared with the data for the $p_{\mathrm{T}}$ range $0.5<p_{\mathrm{T}}<$ $2 \mathrm{GeV}$. The theoretical predictions of the $\rho$ coefficient are based on the nucleon Glauber MC models [13]. Predictions are found to be consistent with the data within the model uncertainties.
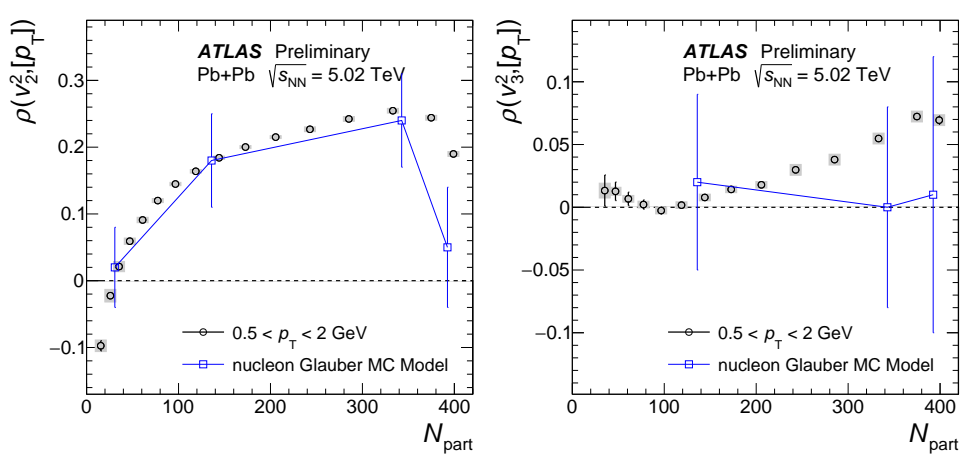

Figure 6: Comparison of the nucleon Glauber model predictions for the modified Pearson's coefficient, $\rho\left(v_{2}^{2},\left[p_{\mathrm{T}}\right]\right)$ (left) $\rho\left(v_{3}^{2},\left[p_{\mathrm{T}}\right]\right)$ (right) for particles with $0.5<p_{\mathrm{T}}<2 \mathrm{GeV}$ [13].

\section{Summary}

This note presents measurements of azimuthal anisotropy of charged particles in $\mathrm{Xe}+\mathrm{Xe}$ collisions at $\sqrt{s_{\mathrm{NN}}}=5.44 \mathrm{TeV}$ in the ATLAS experiment at the LHC. The measurements are performed using an integrated luminosity of $3 \mu \mathrm{b}^{-1}$. The $v_{\mathrm{n}}$ flow harmonics are measured using the SP, 2PC and multi-particle cumulant methods for $\mathrm{n}=2-5$. The measurements are made over wide transverse momentum $\left(0.5<p_{\mathrm{T}}<20 \mathrm{GeV}\right)$ and centrality $(0-80 \%)$ ranges. All harmonics show a similar $p_{\mathrm{T}}$ dependence: first an increase with $p_{\mathrm{T}}$ up to a maximum around $3-4 \mathrm{GeV}$ and then a decrease 
for higher $p_{\mathrm{T}}$. The elliptic flow signal is strongly dependent on the collision centrality and it is the largest in mid-central events (30-50\%). The higher-order harmonics show a weaker centrality dependence, which is consistent with an anisotropy associated with fluctuations in the initial geometry. In the $\mathrm{Xe}+\mathrm{Xe}$ collisions, the normalized symmetric cumulant $\mathrm{nsc}_{2,3}\{4\}$ is found to be negative, reflecting an anti-correlation between $v_{2}$ and $v_{3}$. A strong positive correlation between $v_{2}$ and $v_{4}$ harmonics is indicated by the positive values of $\operatorname{nsc}_{2,4}\{4\}$ and $\operatorname{nac}_{2,4}\{3\}$. The $v_{\mathrm{n}}$ measurements in $\mathrm{Xe}+\mathrm{Xe}$ are compared with the $v_{\mathrm{n}}$ in $\mathrm{Pb}+\mathrm{Pb}$ collisions at $\sqrt{s_{\mathrm{NN}}}=5.02 \mathrm{TeV}$.

The measurement of the modified Pearson's correlation coefficient $\rho$ between the flow harmonics and the per-event mean transverse momentum is performed using $22 \mu \mathrm{b}^{-1}$ of minimumbias 5.02 $\mathrm{TeV} \mathrm{Pb}+\mathrm{Pb}$ data. A strong positive correlation $\rho\left(v_{2}^{2},\left[p_{\mathrm{T}}\right]\right)$ is observed in mid-central and central collisions while negative values are measured for peripheral events. The correlation $\rho\left(v_{3}^{2},\left[p_{\mathrm{T}}\right]\right)$, is found to be weaker. The values of $\rho\left(v_{4}^{2},\left[p_{\mathrm{T}}\right]\right)$ are also positive over the entire centrality range studied. The centrality dependencies have a non-monotonic behaviour in central events, suggesting a change in the nature of the source of the correlations in those events.

The novel measurements can be used to understand the underlying mechanism of QGP dynamics and constrain theoretical models.

This work was supported in part by the National Science Centre, Poland grant 2016/23/B/ST2/00702 and by PL-Grid Infrastructure.

\section{References}

[1] S. Ryu and et al., Phys. Rev. Lett. 115 (2015) 132301, arXiv:1502.01675 [nucl-th].

[2] ATLAS Collaboration, Phys. Rev. C 86 (2012) 014907, arXiv: 1203.3087 [hep-ex] .

[3] ATLAS Collaboration,. ATLAS-CONF-2018-011. http://cds.cern.ch/record/2318870.

[4] ATLAS Collaboration, Phys. Rev. C92 (2015) 034903, arXiv:1504.01289 [hep-ex].

[5] U. Heinz and R. Snellings, Ann. Rev. Nucl. Part. Sci. 63 (2013) 123-151, arXiv: 1301.2826 [nucl-th].

[6] ATLAS Collaboration,. ATLAS-CONF-2018-008. http://cds.cern.ch/record/2318589.

[7] ATLAS Collaboration, JINST 3 (2008) S08003.

[8] STAR Collaboration, Phys.Rev. C66 (2002) 034904, arXiv: nucl-ex/ 0206001.

[9] G. Giacalone and et al., Phys.Rev. C97 (2018) 034904, arXiv:1711.08499 [nucl-th] .

[10] G. Giacalone and et al., Phys.Rev. C94 (2016) 014906, arXiv:1605.08303 [nucl-th] .

[11] J. Jia, M. Zhou, and A. Trzupek, Phys. Rev. C96 (2017) 034906, arXiv: 1701.03830 [nucl-ex].

[12] ATLAS Collaboration, submitted to Phys. Lett. B, arXiv:1807.02012 [nucl-ex].

[13] P. Bozek, Phys. Rev. C93 (2016) 044908 and private communication, arXiv: 1601.04513 [nucl-th].

[14] ATLAS Collaboration, Eur. Phys. J. C74 (2014) 3157, arXiv: 1408.4342 [hep-ex] .

[15] STAR Collaboration, Phys. Rev. C72 (2005) 044902, arXiv: nucl-ex/0504031 [nucl-ex].

[16] ALICE Collaboration, Eur. Phys. J. C74 (2014) 3077, arXiv:1407.5530 [nucl-ex].

[17] A. Mazeliauskas and D. Teaney, Phys. Rev. C93 (2016) 024913, arXiv: 1509.07492 [nucl-th]. 